\title{
A high-throughput contact-hole resolution metric for photoresists: full-process sensitivity study
}

\author{
Christopher N. Anderson ${ }^{a}$ and Patrick P. Naulleau ${ }^{b}$ \\ ${ }^{a}$ Applied Science \& Technology Graduate Group, University of California, Berkeley \\ Berkeley, CA 94720, USA; \\ ${ }^{b}$ Center for X-ray Optics, Lawrence Berkeley National Laboratory \\ 1 Cyclotron Road, Berkeley, CA 94720, USA
}

\begin{abstract}
The ability to accurately quantify the intrinsic resolution of chemically amplified photoresists is critical for the optimization of resists for extreme ultraviolet (EUV) lithography. We have recently reported on two resolution metrics that have been shown to extract resolution numbers consistent with direct observation. In this paper we examine the previously reported contact-hole resolution metric and explore the sensitivity of the metric to potential error sources associated with the experimental side of the resolution extraction process. For EUV exposures at the SEMATECH Berkeley microfield exposure tool, we report a full-process error-bar in extracted resolution of $1.75 \mathrm{~nm}$ RMS and verify this result experimentally.
\end{abstract}

Keywords: Extreme ultraviolet, EUV, lithography, resolution, photoresist

\section{INTRODUCTION}

Extreme ultraviolet (EUV) lithography continues to be the leading candidate for high-volume chip production beyond the 32-nm technology node and has now entered the commercialization phase $[1,2]$. One of the biggest challenges still facing EUV is the development of resists that simultaneously achieve the resolution, sensitivity and line-edge-roughness (LER) requirements for commercialization [3,4]. A large part of the resist development task relies on print-based performance tests to identify resist formulations that meet the demanding specs beyond the 32-nm node. Resist sensitivity and LER are easy to quantify and compare based on direct observation of printing results. The determination of intrinsic resolution, however, is less straightforward.

To address the issue of intrinsic resolution quantification, a variety of methods have been developed including the iso-focal bias [5], LER correlation length [6], modulation transfer function (MTF) [7,8], and contact-hole [9] resolution metrics. Of these four metrics, it has been shown that only the MTF and contact-hole metrics extract resolutions that are consistent with direct observation $[9,10]$. At the present time, the contact-hole resolution metric is the most attractive candidate for high-throughput resist screening owing to the fact that it is the most efficient* $[9]$.

In previous work the error-bars of the contact-hole metric have been determined at EUV wavelengths based on known uncertainties in exposure tool aberrations and focus control that limit the ability to accurately model the aerial image at the wafer surface in an exposure [9]. In this paper we characterize the sensitivity of the contact-hole metric to potential error sources associated with the experimental side of the resolution extraction process. We will explore the following issues: picking the best-focused row from a focus-exposure matrix (FEM), scanning electron microscope (SEM) focus, SEM electron beam dosing, and SEM image analysis. We also perform a full-process reproducibility study to observe how the entire process holds up against the collection of experimental and modeling errors.

Further author information: (Send correspondence to C.N.A.)

C.N.A: E-mail: cnanderson@berkeley.edu, Telephone: 15104865288

P.P.N.: E-mail: pnaulleau@lbl.gov, Telephone: 15104864529

${ }^{*}$ Efficiency is determined by the number of exposure tool use-hours, SEM images, analysis time and modeling support required for resolution extraction, with less time and effort being more efficient. 


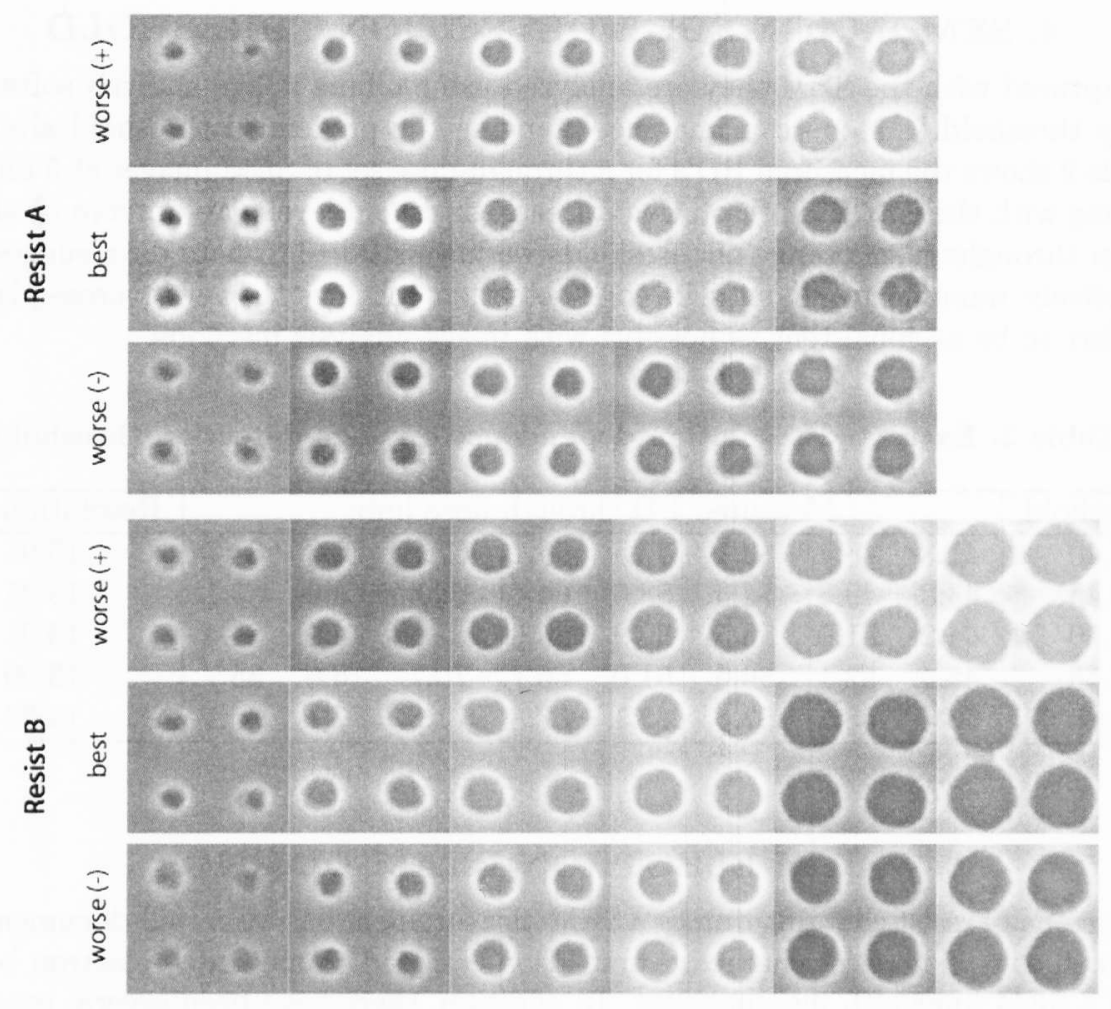

Figure 1. Through-dose SEM images of coded 1:1.5 50-nm dark-field contact-holes printed in resists A (top) and B (bottom) at three focus steps separated by $50 \mathrm{~nm}$ each. The relative dose step between SEM images is 1.32 . The rows labeled 'best' are considered by the authors to be in the best focus.

nm RMS error-bars reported in previous work [9]. For resist A, however, we observe a $2 \mathrm{~nm}$ drop in extracted resolution in the defocused 'worse+' data set as compared to the 'best' data set. Perhaps the explanation is that the 'worse + ' data is in fact the best focus data set and the authors made an incorrect call. Unfortunately we do not have another set of through-focus data on resist A for direct comparison.

Overall the metric proves to be robust even with the ambiguity in experimental data selection. The average of the variances in extracted resolution of each through-focus data set is $1.16 \mathrm{~nm}$, indicating that error-bars associated with determining the best-focus row in the FEM are on the same order as the error-bars associated with aerial image modeling limitations [9]. For resist formulations with small resolutions (i.e., resist A) these data show that cross-platform comparisons could benefit from repeated experimental trials to reduce the measurement uncertainty through statistical averaging.

Table 1. Focus dependence on extracted resolution.

\begin{tabular}{cccc}
\hline Focus $^{a}$ & Resist A & Resist B & Resist H \\
\hline worse + & $7.73(1.33)$ & $21.56(1.68)$ & $12.51(1.20)$ \\
best & $9.90(0.28)$ & $20.50(0.44)$ & $11.53(1.32)$ \\
worse - & $10.39(0.99)$ & $22.70(1.32)$ & $12.33(0.95)$ \\
\hline
\end{tabular}

${ }^{a}$ The focus definitions used here are consistent with those in Figure 1. 
however, each resist behaves a bit differently. At the $4.3 \mu \mathrm{A}$ current setting, resist $\mathrm{C}$ is initially resistant to electron beam exposure showing less than $1 \mathrm{~nm}$ reduction in average PD in the first 120 seconds. Resist D, however, shows more than $6 \mathrm{~nm} \mathrm{PD}$ reduction at the same current setting. In resist $\mathrm{C}$ the $10.6 \mu \mathrm{A}$ current setting is noticeably different than the $4.3 \mu \mathrm{A}$ setting in terms of PD time-evolution. While the $4.3 \mu \mathrm{A}$ setting has a stable and slow initial PD reductuion, the $10.6 \mu \mathrm{A}$ setting shows an increase in PD followed by a quicker roll-off to the steady-state rate. In resist D the changes in SEM emission current are less noticeable; the lower 1.3 $\mu \mathrm{A}$ setting has a slightly lower $(4 \mathrm{~nm}) \mathrm{PD}$ drop in the first 120 seconds and the roll-off trends for both emission currents are very similar. Unfortunately, we do not have $10.6 \mu \mathrm{A}$ data for resist $\mathrm{D}$ for direct comparison.

In both resists we've noticed that increasing SEM emission current changes the measured PD. We believe this effect is the result of an increased signal-to-noise ratio that sharpens the edges of the contact wall in the captured image, changing the line-outs as seen by the image analysis software. We suspect, however, that this effect will not noticeably change extracted resolution as it should be consistent throughout all images within a through-dose set provided the emission current remains fixed. That said, this work shows that it is important to maintain the same emission current throughout each through-dose set of images $\ddagger$. In terms of SEM electron beam dosing, the observed differences between PD time-evolution in resists $\mathrm{C}$ and $\mathrm{D}$ suggest that care should be taken to avoid premature exposure to the electron beam in the desired image area as it affects different resists in different ways and will reduce the credibility of cross-platform comparisons.

\section{SEM FOCUS}

There are many factors that affect the quality of an image captured in a SEM [18]. Assuming that the SEM is well-aligned and is properly corrected for stigma, perhaps the two biggest factors affecting image quality are signal-to-noise ratio and electron beam focus. Figure 3 shows through-SEM-focus images of coded 1:1.5 $50 \mathrm{~nm}$ contacts printed at a dose slightly larger than dose-to-size. The images we show at each SEM focus are subsets of larger raw images containing $>28$ contacts each; reported PD values are the average and variance $(1 \sigma)$ of 20 central contacts in each original image. To avoid SEM electron beam dosing between image captures a different (neighboring) part of the field is imaged at each SEM focus. For calibration purposes we also image seven neighboring field sites at the middle SEM focus and perform a field uniformity test. We find that average and variance of $\mathrm{PD}_{\text {avg }}$ are 60.9 and $0.98 \mathrm{~nm}$, respectively, indicating that the $\approx 5 \mathrm{~nm}$ shift in measured $\mathrm{PD}$ through SEM focus is real.

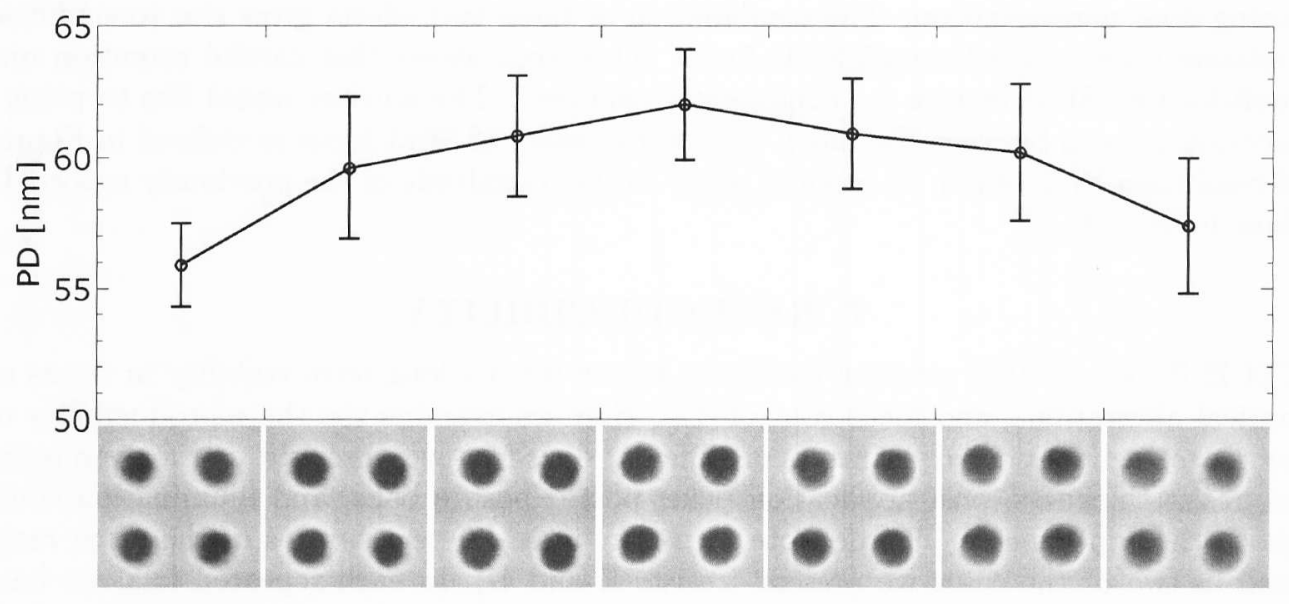

Figure 3. Measured printed diameter (PD) of coded 1:1.5 $50 \mathrm{~nm}$ dark-field contact holes as a function of scanning electron microscope (SEM) focus. The plotted values are the average and standard deviation ( $1 \sigma)$ of 25 neighboring contacts. To avoid SEM electron beam dosing between image captures a different part of the field is imaged at each SEM focus.

\footnotetext{
${ }^{\ddagger}$ We have found that manually controlling the brightness knob on the SEM enables the through-dose set to be captured at one emission current setting while maintaining a reasonable dynamic range throughout the series of images.
} 
Table 3. Reproducibility of the contact-hole resolution metric.

\begin{tabular}{|c|c|c|}
\hline Resist E & Resist F & Resist G \\
\hline 22.14 & 16.71 & 26.56 \\
\hline 20.50 & 16.24 & $25.46^{*}$ \\
\hline $20.88^{*}$ & & \\
\hline \multicolumn{3}{|c|}{ Exposure dates } \\
\hline $12-04-2007$ & $10-25-2007$ & $09-30-2006$ \\
\hline $12-05-2007$ & $10-26-2007$ & $04-06-2007^{*}$ \\
\hline 09-07-2007* & & \\
\hline
\end{tabular}

${ }^{*}$ Resist is from a different resist bottle than the above result(s).

that have been previously reported [9]. These data demonstrate that the error-bars discussed here and in previous work are representative of what we find in practice.

\section{SUMMARY}

In this paper we have examined the sensitivity of the contact-hole resolution metric to several sources of error associated with the experimental side of the resolution extraction process. This work has shown that for EUV exposures at the SEMATECH Berkeley MET printing facility, the contact metric has experimental sources of error that are on the same order as the $1.23 \mathrm{~nm}$ RMS error-bars associated with modeling limitations [9]. Adding the experimental and modeling error-bars in quadrature we determine the full-process error-bar of the contact metric to be $1.75 \mathrm{~nm}$ RMS. The results of the reproducibility study support this conclusion. In repeated trials of resolution extraction for three different resists we find the shift in extracted resolution between trials is at most $1.64 \mathrm{~nm}$ (resist E) and is on average only about $1 \mathrm{~nm}$. The experimental integrity of the contact-hole metric, combined with its low overhead in terms of modeling support and SEM images [9], make it an attractive platform for resolution characterization.

\section{ACKNOWLEDGMENTS}

The authors are greatly indebted to Paul Denham, Ken Goldberg, Brian Hoef, Gideon Jones, and Jerrin Chiu of the Center for X-Ray Optics at Lawrence Berkeley National Laboratory for expert support with the exposure tool as well as the entire CXRO engineering team for building and maintaining the EUV exposure tool. We also acknowledge Tom Wallow, Robert Brainard, and Kim Dean for their support throughout this work. The authors are grateful for support from the NSF EUV Engineering Research Center. This research was also supported by SEMATECH and performed at Lawrence Berkeley National Laboratory using the SEMATECH MET exposure facility at the Advanced Light Source. Lawrence Berkeley National Laboratory is operated under the auspices of the Director, Office of Science, Office of Basic Energy Science, of the US Department of Energy.

\section{REFERENCES}

1. H. Meiling, et. al, "First performance results of the ASML alpha demo tool," Proc. SPIE 6151, (2006).

2. M. Miura, K. Murakami, K. Suzuki, Y. Kohama, Y. Ohkubo, T. Asami, "Nikon EUVL development progress summary," Proc. SPIE 6151, (2006).

3. Stefan Wurm, "EUV Lithography Development in the United States" presented at the 4th International EUVL Symposium Steering Committee, San Diego, CA, November 7-9, 2005, proceedings available from SEMATECH, Austin, TX.

4. B. Wu and A. Kumar, "Extreme ultraviolet lithography: A review," J. Vac. Sci. Technol. B 25(6), Nov/Dec 2007.

5. G. M. Schmid, M. D. Stewart, C. Wang, B. D. Vogt, M. Vivek, E. K. Lin, C G Willson, "Resolution limitations in chemically amplified photoresist systems," Proc. of SPIE 5376, 333-342, (2004). 